\title{
Devastating yet treatable complication of tuberculous meningitis: the resistant TB abscess
}

\author{
J. F. Salomão
}

Published online: 27 February 2009

(C) Springer-Verlag 2009

Neurotuberculosis (NTB) is a persistent disease in developing countries and its morbidity and mortality remain high in children, especially when there is a delay in diagnosis. NTB may be present mainly as tuberculous meningitis (TBM) or tuberculomas. TBM is the most common form of the disease in children and characteristically takes the form of a basal exudate seen in CT scan as a hyperdense lesion as shown by the authors. In this form, hydrocephalus is a common and often troublesome complication. Tuberculomas are less frequently seen and appear as isolated or multiple granulomatous lesions containing caseous material. Tuberculous abscesses (TBA) are encapsulated collections of pus containing viable acid-fast bacilli [3], are a rare form of NTB, and must be differentiated from tubercular granuloma with central caseation or necrosis [7]. The case of a patient with TBM who initially responded to a standard four-drug regimen plus corticosteroids, but subsequently deteriorated, is presented.

This unusual evolution can be seen in two situations: paradoxical progression or multidrug-resistant tuberculosis (MDR-TBM). In the first case, lesions increase in size and number despite the drug susceptibility and adequate adherence to treatment $[1,6]$. Late deterioration is ascribed

This commentary refers to the article doi: 10.1007/s00381-009-0808-8.

J. F. Salomão $(\triangle)$

Section of Pediatric Neurosurgery, Department of Pediatric

Surgery, Fernandes Figueira Institute, OswaldoCruz Foundation

(M.S.-Fiocruz),

Av. Rui Barbosa, 716,

22250-020 Rio de Janeiro, RJ, Brazil

e-mail: jfsalomao@terra.com.br to enlargement of preexisting lesions, development of new lesions, or hydrocephalus. These lesions usually resolve themselves over time, without changing the drugs regimen, although in some cases surgical excision is recommended $[4,7]$. These phenomena can develop for even up to 1 year $[1,4]$. Multidrug-resistant tuberculosis has become a worldwide problem and is often associated with a grave outcome. It is defined as resistance to at least isoniazid and rifampicin. It can be due to an "acquired" resistance or to reinfection with MDR strains, especially if in the household there is an adult source with drug-resistant tuberculosis [8]. More information regarding the bacteriological and epidemiological data would be useful in this case.

There is no mention about HIV screening of this patient. It is known that AIDS-related tuberculosis is more prevalent and the incidence of MDR-TBM in HIV infection is very high. Additionally, in the AIDS era, paradoxical reactions have been related to concurrent administration of highly active antiretroviral therapy with antituberculous therapy [5]. Moreover, in the case of associated HIV infection, a differential diagnosis with other ring-enhanced lesions is mandatory, especially when dealing with refractory cases.

Concerning the brain atrophy and the diffuse ischemic lesion, it is known that basal exudates seen in NTB can entrap and even occlude arteries of the circle of Willis and inflammatory vasculitis can induce focal and diffuse brain changes as well [2]. It is not usual for patients with status epilepticus present with such a severe anoxic lesion and in the present case one must consider TBM-induced vascular changes at least as an important adjuvant factor.

We agree with the authors that ring-enhancing lesions that do not respond to specific therapy should be explored in order to reduce complications, improve the prognosis, and eventually make evident other unexpected intracranial lesions. 


\section{References}

1. Afghani B, Lieberman JM (1994) Paradoxical enlargement or development of intracranial tuberculomas during therapy: case report and review. Clin Infect Dis 19:1092-1099

2. Dastur DK, Manghani DK, Udani PM (1995) Pathology and pathogenetic mechanisms in neurotuberculosis. Radiol Clin North Am 33:733-752

3. Kumar R, Pandey CK, Bose N, Sahay S (2002) Tuberculous brain abscess: clinical presentation, pathophysiology and treatment (in children). Childs Nerv Syst 18:118-123

4. Kumar R, Prakash M, Sanjeev J (2006) Paradoxical response to chemotherapy in neurotuberculosis. Pediatr Neurosurg 42:214-222
5. Narita M, Ashkin D, Hollender ES, Pitchenik AE (1998) Paradoxal worsening of tuberculosis following antiretroviral therapy in patients with AIDS. Amer J Resp Crit Care Med 158:157-161

6. Rao GP, Nadh BR, Hemaratnan A, Srinivas TV, Reddy PK (1995) Paradoxical progression of tuberculous lesions during chemotherapy of central nervous system tuberculosis. J Neurosurg 83:359362

7. Ravenscroft A, Schoeman JF, Donald PR (2001) Tuberculous granulomas in childhood tuberculous meningitis: radiological features and course. J Trop Pediatr 47:5-12 Kumar abscess

8. Schaaf HS, Shean K, Donald PR (2003) Culture confirmed multidrug resistant tuberculosis: diagnostic delay, clinical features, and outcome. Arch Dis Child 88:1106-1111 